\begin{tabular}{|c|l|}
\hline Title & ILL-POSEDNESS EXAMPLES FOR THE QUA SI-GEOST ROPHIC AND THE EULER EQUATIONS \\
\hline Author(s) & MISIOLEK, GERA RD; Y ONEDA, TSUY OSHI \\
\hline Citation & Hokkaido University Preprint Series in Mathematics, 996, 1-9 \\
\hline Issue Date & 2012-2-2 \\
\hline DOI & 10.14943/84143 \\
\hline Doc URL & http://hdl.handle.net/2115/69802 \\
\hline Type & bulletin (article) \\
\hline File Information & pre996.pdf \\
\hline
\end{tabular}

Instructions for use 


\title{
ILL-POSEDNESS EXAMPLES FOR THE QUASI-GEOSTROPHIC AND THE EULER EQUATIONS
}

\author{
GERARD MISIOŁEK AND TSUYOSHI YONEDA
}

\begin{abstract}
We construct examples of ill-posedness of solutions of the 2D QG and the 3D Euler equations in the Besov and the logarithmic Lipschitz spaces.
\end{abstract}

\section{INTRODUCTION}

A Cauchy problem is said to be (locally) well-posed in a Banach space $X$ according to Hadamard if for any data in $X$ there exists $T>0$ and a unique solution in the space $C([0, T), X)$ which depends at least continuously on the data. In particular, solutions describe continuous curves in $X$ at least for a short time. The problem is said to be ill-posed in $X$ if it is not well-posed in the above sense.

A number of different mechanisms can lead to ill-posedness. They range from the loss of regularity properties of the solution map to non-uniqueness to finite-time blowup. In this paper we focus on two problems: a failure of continuity in time of the solution map of the 2D quasi-geostrophic equation in Besov spaces and a loss of regularity of solutions to the 3D Euler equations in the space of logarithmic Lipschitz functions.

The Cauchy problem for the quasi-geostrophic equation has the form

$$
\begin{aligned}
& \partial_{t} \theta+u \cdot \nabla \theta+\kappa(-\Delta)^{\alpha / 2} \theta=0 \\
& \theta(0, x)=\theta_{0}(x), \quad x \in \mathbb{T}^{2}=\mathbb{R}^{2} / 2 \pi \mathbb{Z}^{2}
\end{aligned}
$$

where $\kappa \geq 0, \alpha>0$ and

$$
u=\left(R_{2} \theta,-R_{1} \theta\right), \quad \widehat{R_{j} \theta}(\xi)=-i \frac{\xi_{j}}{|\xi|} \hat{\theta}(\xi), \quad \xi_{j} \in \mathbb{Z} \quad(j=1,2) .
$$

This equation was introduced in [CMT] as a two-dimensional model of the 3D Euler equations. The subcritical $(\alpha>1)$ and critical $(\alpha=1)$ cases are rather well understood while local well-posedness in Besov spaces for the supercritical case $(\alpha<1)$ can be found in $[\mathrm{HK}]$.

In $[\mathrm{KNV}]$ the authors observed that if $\theta_{0} \in L^{p}\left(\mathbb{T}^{2}\right)$ with $1<p<\infty$ then the solution of (1.1) in the critical case satisfies $\lim _{t \rightarrow 0^{+}}\left\|\theta(t)-\theta_{0}\right\|_{L^{p}}=0$. Our first result shows that this property fails in certain Besov spaces for $0<\alpha<1$. The construction uses elementary properties of certain lacunary Fourier series. A strategy of this type was applied recently in [CS] to the Euler and Navier-Stokes equations.

Date: January 30, 2012.

1991 Mathematics Subject Classification. 35Q35. 
Well-posedness results for the Euler equations of hydrodynamics

$$
\begin{aligned}
& \partial_{t} u+\nabla_{u} u=-\nabla p \\
& \operatorname{div} u=0 \\
& u(0, x)=u_{0}(x), \quad x \in \mathbb{T}^{3} \text { or } \mathbb{R}^{3}
\end{aligned}
$$

were obtained already in the 1920s and 1930s. A modern exposition can be found in the monograph $[\mathrm{MB}]$. On the other hand, the first rigorous studies of ill-posedness such as the construction of non-unique or energy-decreasing solutions are more recent, see $[\mathrm{Sch}]$ or $[\mathrm{Shn}]$.

Our second result provides an example of an instantaneous loss of smoothness of solutions of (1.4) in the scale of logarithmic Lipschitz spaces. Such examples were first constructed in $[\mathrm{BT}]$ in the Hölder-Zygmund spaces using the shear flow solution of DiPerna and Majda.

\section{The supercritical QG equation in $B_{p, \infty}^{s}\left(\mathbb{T}^{2}\right)$}

We recall some basic harmonic analysis. Given a smooth radial bump function $\varphi$ on $\mathbb{R}^{2}$ supported in the unit ball and equal to 1 on the ball of radius $1 / 2$, we set

$$
\varphi_{0}(\xi)=\varphi\left(2^{-1} \xi\right)-\varphi(\xi) \quad \text { and } \quad \varphi_{k}(\xi)=\varphi_{0}\left(2^{-k} \xi\right) \quad \forall k \geq 0 .
$$

Each $\varphi_{k}$ is supported in the shell $\left\{2^{k-1} \leq|\xi| \leq 2^{k+1}\right\}$ with $\varphi_{k}(\xi)=1$ when $|\xi|=2^{k}$. We also set $\varphi_{-1}=\varphi$ and, for any periodic distribution $f$ on the torus, define the frequency restriction operators by

$$
\widehat{\Delta_{k} f}(\xi)=\varphi_{k}(\xi) \hat{f}(\xi), \quad \forall k \geq-1
$$

to obtain the usual Littlewood-Paley decomposition of $f$

$$
f=\sum_{k \geq-1} \Delta_{k} f \quad \text { where } \quad \Delta_{k} f(x)=\sum_{\xi \in \mathbb{Z}^{2}} \varphi_{k}(\xi) \hat{f}(\xi) e^{i\langle\xi, x\rangle}, \quad x \in \mathbb{T}^{2} .
$$

For any $s \in \mathbb{R}$ and $1 \leq p, q \leq \infty$ the Besov space $B_{p, q}^{s}\left(\mathbb{T}^{2}\right)$ is defined as consisting of the periodic distributions for which the norm

$$
\|f\|_{B_{p, q}^{s}}=\left\{\begin{array}{llr}
\left(\sum_{k \geq-1} 2^{k s q}\left\|\Delta_{k} f\right\|_{L^{p}}^{q}\right)^{1 / q} & \text { if } & 1 \leq q<\infty \\
\sup _{k \geq-1} 2^{k s}\left\|\Delta_{k} f\right\|_{L^{p}} & \text { if } & q=\infty
\end{array}\right.
$$

is finite. Two special cases of interest are the Sobolev space $H^{s}\left(\mathbb{T}^{2}\right)=B_{2,2}^{s}\left(\mathbb{T}^{2}\right)$ and the Hölder-Zygmund class $\mathcal{C}^{s}\left(\mathbb{T}^{2}\right)=B_{\infty, \infty}^{s}\left(\mathbb{T}^{2}\right)$.

Our main result in this section is

Theorem 2.1. Let $0<\alpha<1$. There exists $\theta_{0} \in B_{p, \infty}^{s}\left(\mathbb{T}^{2}\right)$ such that the corresponding (weak) solution $\theta$ of the Cauchy problem (1.1)-(1.2) satisfies

$$
\lim _{t \rightarrow 0^{+}}\left\|\theta(t)-\theta_{0}\right\|_{B_{p, \infty}^{s}}>0
$$


for any $s>0$ and any $2 \leq p \leq \infty$.

Proof. Consider the function defined by the lacunary series ${ }^{1}$

$$
\theta_{0}\left(x_{1}, x_{2}\right)=\cos x_{2}+\sum_{k=1}^{\infty} 2^{-k s} \cos 2^{k} x_{1} .
$$

It is not difficult to check that $\theta_{0} \in B_{p, \infty}^{s}\left(\mathbb{T}^{2}\right)$ for any $1 \leq p \leq \infty$. Next, consider the following sequence of Fourier frequencies

$$
\xi_{k}=\left(2^{k}, 1\right) \quad \text { for } \quad k=1,2 \ldots
$$

Using (2.3) and the formula for the Riesz transforms in (1.3) we can compute explicitly the corresponding Fourier term

$$
\begin{aligned}
\vartheta_{k}:=\widehat{u_{0} \nabla \theta_{0}}\left(\xi_{k}\right) & =\left(\operatorname{div}\left(\theta_{0} u_{0}\right)\right)\left(\xi_{k}\right)=i\left\langle\xi_{k}, \hat{\theta}_{0} * \hat{u}_{0}\left(\xi_{k}\right)\right\rangle \\
& =\sum_{\eta \in \mathbb{Z}^{2}} \hat{\theta}_{0}\left(\xi_{k}-\eta\right) \hat{\theta}_{0}(\eta) \frac{\xi_{k, 1} \eta_{2}-\xi_{k, 2} \eta_{1}}{|\eta|} \\
& =\frac{1}{4} 2^{k(1-s)}\left(1-2^{-k}\right) .
\end{aligned}
$$

Let $e^{-t(-\Delta)^{\alpha}}$ denote the semigroup generated by the fractional Laplacian. Using the partial Fourier transform in the space variables we can rewrite equation (1.1) in an integral form and for any $t \geq 0$ obtain the relation

$$
\hat{\theta}\left(t, \xi_{k}\right)=-\int_{0}^{t} e^{-(t-\tau)\left|\xi_{k}\right|^{\alpha}} \widehat{u \cdot \nabla \theta}\left(\tau, \xi_{k}\right) d \tau,
$$

since $\hat{\theta}_{0}\left(\xi_{k}\right)=0$ by construction.

We claim that for any $t>0, s>0$ and $2 \leq p \leq \infty$ there is an estimate

$$
\begin{aligned}
& \left|\hat{\theta}\left(t, \xi_{k}\right)+\int_{0}^{t} e^{-(t-\tau)\left|\xi_{k}\right|^{\alpha}} \vartheta_{k} d \tau\right| \leq \\
& \quad \leq C 2^{k(1-s)} \frac{1-e^{-t\left|\xi_{k}\right|^{\alpha}}}{4\left|\xi_{k}\right|^{\alpha}} \sup _{0 \leq \tau \leq t}\left(\|\theta(\tau)\|_{B_{p, \infty}^{s}}+\left\|\theta_{0}\right\|_{B_{p, \infty}^{s}}\right) \sup _{0 \leq \tau \leq t}\left\|\theta(\tau)-\theta_{0}\right\|_{B_{p, \infty}^{s}}
\end{aligned}
$$

where $C>0$ is a constant independent of $k$.

To show this observe that if $k \geq 1$ then $2^{k}<\left|\xi_{k}\right| \leq 2^{k} \sqrt{2}$ so that in particular we have a lower bound

$$
\varphi_{k}\left(\xi_{k}\right) \geq \varphi(1,1 / 2)=c>0 .
$$

\footnotetext{
${ }^{1}$ We refer to [Zyg] for properties of lacunary series of this type; see also [Tai].
} 
Using (2.8) together with (2.6) we first bound the left side of (2.7) by

$$
\begin{aligned}
\leq \frac{1}{c} \mid \int_{0}^{t} e^{-(t-\tau)\left|\xi_{k}\right|^{\alpha}} \varphi_{k}\left(\xi_{k}\right)\left(\left(u-u_{0}\right) \cdot \nabla \theta \hat{)}\left(\tau, \xi_{k}\right) d \tau \mid\right. \\
+\frac{1}{c} \mid \int_{0}^{t} e^{-(t-\tau)\left|\xi_{k}\right|^{\alpha}} \varphi_{k}\left(\xi_{k}\right)\left(u_{0} \cdot \nabla\left(\theta-\theta_{0}\right) \hat{)}\left(\tau, \xi_{k}\right) d \tau \mid\right.
\end{aligned}
$$

which, using the relation $\widehat{\Delta_{k} f}=\varphi_{k} \hat{f}$, can be estimated further by

$$
\lesssim \int_{0}^{t} e^{-(t-\tau)\left|\xi_{k}\right|^{\alpha}}\left\|\Delta_{k}\left(\left(u-u_{0}\right) \cdot \nabla \theta\right)\right\|_{L^{1}} d \tau+\int_{0}^{t} e^{-(t-\tau)\left|\xi_{k}\right|^{\alpha}}\left\|\Delta_{k}\left(u_{0} \cdot \nabla\left(\theta-\theta_{0}\right)\right)\right\|_{L^{1}} d \tau \text {. }
$$

In order to bound the $L^{1}$ norms in the above integrands we use the following lemma.

Lemma 2.2. For any $k \geq 1$ and $s>0$ we have

$$
\left\|\Delta_{k}(v \cdot \nabla \phi)\right\|_{L^{1}\left(\mathbb{T}^{2}\right)} \lesssim 2^{k(1-s)}\left(\|\phi\|_{L^{p_{1}}}\|v\|_{B_{p_{2}, q}^{s}}+\|\phi\|_{B_{p_{3}, q}^{s}}\|v\|_{L^{p_{4}}}\right)
$$

where $1 \leq p_{1}, p_{4}, q \leq \infty$ and $1 / p_{1}+1 / p_{2}=1 / p_{3}+1 / p_{4}=1$ and $v$ is divergence-free.

Proof. Since the Littlewood-Paley projections commute with derivatives and $\operatorname{div} v=0$ we have by Bernstein's inequality

$$
\left\|\Delta_{k}(v \cdot \nabla \phi)\right\|_{L^{1}}=\left\|\Delta_{k} \operatorname{div}(\phi v)\right\|_{L^{1}} \lesssim 2^{k} \sum_{j}\left\|\Delta_{k}\left(\phi v^{j}\right)\right\|_{L^{1}}
$$

and the lemma is now an immediate consequence of the estimate for pointwise products of Besov functions

$$
\|f g\|_{B_{1, q}^{s}} \lesssim\|f\|_{L^{p_{1}}}\|g\|_{B_{p_{2}, q}^{s}}+\|f\|_{B_{p_{3}, q}^{s}}\|g\|_{L^{p_{4}}}
$$

which is a straightforward generalization of the analogous inequality for $B_{p, p}^{s}$ Sobolev norms (see e.g. [Tay], Chapter 2).

Using Lemma 2.2, taking supremum over $[0, t]$ and integrating the exponential factor, the left hand side of (2.7) is bounded by

$$
\begin{aligned}
& \lesssim 2^{k(1-s)} \frac{1-e^{-t\left|\xi_{k}\right|^{\alpha}}}{\left|\xi_{k}\right|^{\alpha}} \sup _{0 \leq \tau \leq t}\left\{\|\theta\|_{L^{p_{1}}}\left\|u-u_{0}\right\|_{B_{p_{2}, \infty}^{s}}+\|\theta\|_{B_{p_{3}, \infty}^{s}}\left\|u-u_{0}\right\|_{L^{p_{4}}}\right. \\
& \left.+\left\|\theta-\theta_{0}\right\|_{L^{p_{1}}}\left\|u_{0}\right\|_{B_{p_{2}, \infty}^{s}}+\left\|\theta-\theta_{0}\right\|_{B_{p_{3}, \infty}^{s}}\left\|u_{0}\right\|_{L^{p_{4}}}\right\} .
\end{aligned}
$$

Observe that since $2 \leq p \leq \infty$ we can always arrange so that $p_{1}=p_{3}=p$ while the corresponding conjugate exponents satisfy $1 \leq p_{2}=p_{4} \leq 2 \leq p$.

Since the Riesz transforms $R_{j}$ are bounded in $L^{r}$ with any $1<r<\infty$ it is not difficult to show that $\|u\|_{B_{r, q}^{s}} \lesssim\|\theta\|_{B_{r, q}^{s}}$ for any $1 \leq q \leq \infty$. In fact, we have

Lemma 2.3. The operators $R_{j}(j=1,2)$ are bounded from $B_{p, q}^{s}\left(\mathbb{T}^{2}\right)$ into itself for any $s>0$ and any $1 \leq p, q \leq \infty$.

Proof. See [Tai], Part II. 
Applying Lemma 2.3 together with standard embedding results for Besov spaces we can now dominate the terms appearing inside the supremum in expression (2.10) by the following sum

$$
\begin{aligned}
\|\theta\|_{B_{p_{1}, \infty}^{s}}\left\|\theta-\theta_{0}\right\|_{B_{p_{2}, \infty}^{s}}+\|\theta\|_{B_{p_{3}, \infty}^{s}}\left\|\theta-\theta_{0}\right\|_{B_{p_{4}, \infty}^{s}}+\left\|\theta-\theta_{0}\right\|_{B_{p_{1}, \infty}^{s}}\left\|\theta_{0}\right\|_{B_{p_{2}, \infty}^{s}} & +\left\|\theta-\theta_{0}\right\|_{B_{p_{3}, \infty}^{s}}\left\|\theta_{0}\right\|_{B_{p_{4}, \infty}^{s}}
\end{aligned}
$$

where $p_{1}, p_{2}, p_{3}$ and $p_{4}$ are chosen as before. Furthermore, using monotonicity of periodic Besov spaces with respect to the (primary) integrability index each of the norms in the above expression can be bounded by the corresponding $B_{p, \infty}^{s}$ norm since by our choice we have $p=\max _{1 \leq j \leq 4} p_{j}$.

Combining these inequalities we can now estimate (2.10) by

$$
\lesssim 2^{k(1-s)} \frac{1-e^{-t\left|\xi_{k}\right|^{\alpha}}}{\left|\xi_{k}\right|^{\alpha}} \sup _{0 \leq \tau \leq t}\left(\|\theta(\tau)\|_{B_{p, \infty}^{s}}+\left\|\theta_{0}\right\|_{B_{p, \infty}^{s}}\right) \sup _{0 \leq \tau \leq t}\left\|\theta(\tau)-\theta_{0}\right\|_{B_{p, \infty}^{s}}
$$

which establishes our claim.

We next derive a lower bound for the expression on the left side of inequality (2.7). This is immediate from (2.5) and the triangle inequality

$$
\begin{aligned}
\left|\hat{\theta}\left(t, \xi_{k}\right)+\int_{0}^{t} e^{-(t-\tau)\left|\xi_{k}\right|^{\alpha}} \vartheta_{k} d \tau\right| & \geq\left|\int_{0}^{t} e^{-(t-\tau)\left|\xi_{k}\right|^{\alpha}} \vartheta_{k} d \tau\right|-\left|\hat{\theta}\left(t, \xi_{k}\right)\right| \\
& =\frac{1-e^{-t\left|\xi_{k}\right|^{\alpha}}}{4\left|\xi_{k}\right|^{\alpha}} 2^{k(1-s)}\left(1-2^{-k}\right)-\left|\hat{\theta}\left(t, \xi_{k}\right)\right| .
\end{aligned}
$$

From (2.11) and (2.7) we thus obtain a lower bound on the size of the Fourier coefficient of $\theta(t, x)$ at any $t>0$ and $\xi_{k}$ namely

$$
\left|\hat{\theta}\left(t, \xi_{k}\right)\right| \geq
$$

$\geq \frac{1-e^{-t\left|\xi_{k}\right|^{\alpha}}}{4\left|\xi_{k}\right|^{\alpha}} 2^{k(1-s)}\left(1-2^{-k}-C\left(\sup _{0 \leq \tau \leq t}\|\theta(\tau)\|_{B_{p, \infty}^{s}}+\left\|\theta_{0}\right\|_{B_{p, \infty}^{s}}\right) \sup _{0 \leq \tau \leq t}\left\|\theta(\tau)-\theta_{0}\right\|_{B_{p, \infty}^{s}}\right)$

where $k=1,2 \ldots$ and $C$ is independent of $k$.

We now proceed as follows. Suppose that (2.2) does not hold for some $s>0$ and $2 \leq p \leq \infty$. Pick $0<t_{0} \leq t$ such that

$$
\left\|\theta(\tau)-\theta_{0}\right\|_{B_{p, \infty}^{s}}<\min \left(1, \frac{1}{2 C\left(1+2\left\|\theta_{0}\right\|_{B_{p, \infty}^{s}}\right)}\right)
$$


for all $0 \leq \tau \leq t_{0}$. With these choices inequality (2.12) yields

$$
\begin{aligned}
\left|\hat{\theta}\left(t_{0}, \xi_{k}\right)\right| & \geq \\
\geq \frac{1-e^{-t_{0}\left|\xi_{k}\right|^{\alpha}}}{4\left|\xi_{k}\right|^{\alpha}} 2^{k(1-s)}(1 & \left.-2^{-k}-C\left(\sup _{0 \leq \tau \leq t_{0}}\left\|\theta-\theta_{0}\right\|_{B_{p, \infty}^{s}}+2\left\|\theta_{0}\right\|_{B_{p, \infty}^{s}}\right) \sup _{0 \leq \tau \leq t_{0}}\left\|\theta-\theta_{0}\right\|_{B_{p, \infty}^{s}}\right) \\
& \geq \frac{1-e^{-t_{0}\left|\xi_{k}\right|^{\alpha}}}{8\left|\xi_{k}\right|^{\alpha}} 2^{k(1-s)}-\frac{1-e^{-t_{0}\left|\xi_{k}\right|^{\alpha}}}{4\left|\xi_{k}\right|^{\alpha}} 2^{-k s} \\
& \geq \frac{1}{4}\left(\left(1-e^{-t_{0} 2^{k \alpha}}\right) 2^{k(1-s-\alpha)-2}-2^{-k s}\right)
\end{aligned}
$$

for any $k \geq 1$ which together with (2.8) and $2 \leq p \leq \infty$ implies

$$
\begin{aligned}
\left\|\theta\left(t_{0}\right)\right\|_{B_{p, \infty}^{s}} & \geq \sup _{k \geq 1}\left(2^{k s}\left\|\Delta_{k} \theta\left(t_{0}\right)\right\|_{L^{2}}\right) \geq \sup _{k \geq 1}\left(2^{k s}\left|\hat{\theta}\left(t_{0}, \xi_{k}\right)\right| \varphi_{k}\left(\xi_{k}\right)\right) \\
& >c \sup _{k \geq 1}\left(2^{k s}\left|\hat{\theta}\left(t_{0}, \xi_{k}\right)\right|\right)=\infty
\end{aligned}
$$

provided that $0<\alpha<1$.

The following is an immediate corollary.

Corollary 2.4. The conclusion of Theorem 2.1 also holds for weak solutions in $\mathcal{C}^{s}\left(\mathbb{T}^{2}\right)$ with $0<\alpha<1$ and $s>0$.

\section{Ill-POSEDNESS OF EULER EQUATIONS IN LOG-LIPSCHITZ SPACES}

There are relatively few non-trivial solutions of the Euler equations (1.4) that are known explicitly. Investigating existing examples remains therefore very useful. One such example is the shear flow of DiPerna and Majda

$$
u(t, x)=\left(g\left(x_{2}\right), 0, f\left(x_{1}-t g\left(x_{2}\right)\right)\right), \quad x=\left(x_{1}, x_{2}, x_{3}\right)
$$

used recently in [BT] to construct a (weak) solution with initial data in $\mathcal{C}^{\alpha}$ which does not belong to $\mathcal{C}^{\beta}$ for any $1>\beta>\alpha^{2}$ and any $t>0$. The authors also observed that a similar construction works in the Zygmund space $B_{\infty, \infty}^{1}$ and the Triebel-Lizorkin space $F_{\infty, 2}^{1}$. Clearly, if $f$ and $g$ are in $\mathcal{C}^{1+\alpha}$ with $0<\alpha<1$ then the shear flow satisfies the equations and remains in $\mathcal{C}^{1+\alpha}$ for all time. In fact, since by a result of $[\mathrm{PP}]$ the problem (1.4)-(1.6) is known to be locally well-posed in the Besov space $B_{\infty, 1}^{1}$, examples like (3.1) point to $C^{1}$ as the critical space

$$
\mathcal{C}^{1+\alpha} \subset B_{\infty, 1}^{1} \subset C^{1} \subset F_{\infty, 2}^{1} \subset B_{\infty, \infty}^{1} \subset \mathcal{C}^{\beta} \quad(0<\alpha, \beta<1)
$$

in which the Cauchy problem for the 3D Euler equations is studied.

Consider the scale of logarithmic Lipschitz spaces $L L_{\alpha}\left(\mathbb{R}^{n}\right)$ defined for any $\alpha \geq 0$ as consisting of all continuous functions such that

$$
\|f\|_{L L_{\alpha}}=\|f\|_{\infty}+\sup _{0<|x-y|<1 / 2} \frac{|f(x)-f(y)|}{|x-y||\log | x-\left.y\right|^{\alpha}}<\infty .
$$


Observe that $L L_{0}$ and $L L_{1}$ are the usual spaces of Lipschitz and log-Lipschitz continuous functions respectively and moreover

$$
\text { Lip } \subset L L_{\alpha} \subset \operatorname{logLip} \quad \text { and } \quad C^{1} \subset \operatorname{Lip} \subset F_{\infty, 2}^{1} \subset B_{\infty, \infty}^{1} \subset \log \operatorname{Lip} \subset \mathcal{C}^{\alpha}
$$

for any $0<\alpha<1$.

Our goal in this note is to sharpen the ill-posedness result in the Hölder-Zygmund spaces. Its relation to the counterexample in $F_{\infty, 2}^{1}$ remains somewhat obscure, see Remark 3.2 below and (3.2).

Theorem 3.1. The 3D Euler equation is not well-posed in the space $L L_{\alpha}$ for any $0<\alpha \leq 1$. In fact, there exist shear flow solutions $u_{\alpha}(t)$ of (1.4) with initial data in $L L_{\alpha}$ such that

(1) if $1 / 2 \leq \alpha \leq 1$, then $u_{\alpha}(t) \notin L L_{\beta}$ for any $0<\beta<1$

(2) if $0<\alpha<1 / 2$, then $u_{\alpha}(t) \in L L_{\beta} \Longleftrightarrow 2 \alpha \leq \beta<1$

for any $t>0$.

Proof. The argument relies on the properties of the superposition operator. ${ }^{2}$ The first step is a straightforward modification of the original construction in [BT].

Fix $t>0$ and let $0 \leq \rho \leq 1$ be a smooth cutoff function on $\mathbb{R}$ with compact support which is equal to 1 on an interval $(-\delta, \delta)$ for some $\delta>0$. Given $0<\alpha \leq 1$ consider the shear flow $u_{\alpha}(t)$ defined as in (3.1) where

$$
f(y)=g(y)=\rho(y)|y||\log | y||^{\alpha}, \quad y \in \mathbb{R} .
$$

It is easy to see that $u_{\alpha}(0, x)=\left(f\left(x_{2}\right), 0, f\left(x_{1}\right)\right)$ belongs to $L L_{\alpha}$. On the other hand, for any $t>0$ we check that $\left\|u_{\alpha}(t)\right\|_{\infty}<\infty$. Furthermore, if $x_{1}=0$ then for any $\left|x_{2}\right|<\delta$ the third component satisfies

$$
\begin{aligned}
u_{\alpha, 3}\left(t, 0, x_{2}, x_{3}\right) & =f\left(-t f\left(x_{2}\right)\right)=\rho\left(-t f\left(x_{2}\right)\right)\left|t f\left(x_{2}\right)\right||\log | t f\left(x_{2}\right)||^{\alpha} \\
& \simeq \rho\left(-t\left|x_{2}\right||\log | x_{2}||^{\alpha}\right) t\left|x_{2}\right||\log | x_{2}||^{\alpha}\left|\log \left(t|y||\log | y||^{\alpha}\right)\right|^{\alpha} \\
& \simeq t\left|x_{2}\right||\log | x_{2}||^{\alpha}\left|\log \left(t\left|x_{2}\right||\log | x_{2}||^{\alpha}\right)\right|^{\alpha}
\end{aligned}
$$

provided that $\delta>0$ is chosen sufficiently small, because we have that $\left|x_{2}\right||\log | x_{2}||^{\alpha} \rightarrow 0$ whenever $\left|x_{2}\right| \rightarrow 0$. For simplicity, in what follows we set $t=1$ and denote the right side above by $r_{\alpha}\left(x_{2}\right)$.

Let $0<\alpha \leq \beta \leq 1$. To prove the theorem we need to estimate the second term in (3.3) of the $L L_{\beta}$ norm of $u_{\alpha}(t)$. For this purpose it will be sufficient to examine the behaviour of $r_{\alpha}\left(x_{2}\right)$ around $x_{2}=0$ and hence to consider the quotient

$$
\frac{\left|r_{\alpha}\left(x_{2}\right)-r_{\alpha}(0)\right|}{\left|x_{2}\right||\log | x_{2}||^{\beta}}=\frac{\left|r_{\alpha}\left(x_{2}\right)\right|}{\left|x_{2}\right||\log | x_{2}||^{\beta}}=|\log | x_{2}||^{\alpha-\beta}\left|\log \left(\left|x_{2}\right||\log | x_{2}||^{\alpha}\right)\right|^{\alpha}
$$

\footnotetext{
${ }^{2}$ It cannot therefore be expected to produce counterexamples in spaces like Lip although it should work in any Lipschitz-type space characterized by a concave modulus of continuity.
} 
as $\left|x_{2}\right| \rightarrow 0$. Setting $\lambda=\log \left|x_{2}\right|$ in the expression on the right hand side and computing the limit we obtain

$$
|\lambda|^{\alpha-\beta}|\lambda+\alpha \log | \lambda||^{\alpha}=|\lambda|^{2 \alpha-\beta}\left|1-\alpha \frac{\log |\lambda| \mid}{|\lambda|}\right|^{\alpha} \underset{\lambda \searrow-\infty}{\longrightarrow}\left\{\begin{array}{cc}
+\infty & \text { if } 2 \alpha>\beta \\
1 & \text { if } 2 \alpha=\beta \\
0 & \text { if } 2 \alpha<\beta
\end{array}\right.
$$

which establishes (1) and (2). In particular, it follows that $u_{\alpha}(t) \notin L L_{\alpha}$ for any $t>0$ and any $0<\alpha \leq 1$.

Remark 3.2. In $[\mathrm{EH}]$ the authors showed that

$$
B_{\infty, q}^{1} \subset L L_{\alpha} \Longleftrightarrow \alpha \geq 1-1 / q \quad \text { where } 1<q \leq \infty .
$$

Since the result in (3.4) is sharp and since $B_{\infty, 2}^{1} \subset F_{\infty, 2}^{1}$ we see that $F_{\infty, 2}^{1} \nsubseteq L L_{\alpha}$ whenever $\alpha<1 / 2$. It follows that Theorem 3.1 cannot be directly compared with the counterexample in $F_{\infty, 2}^{1}$ described in $[\mathrm{BT}]$.

Remark 3.3. It is not difficult to check that a suitable modification of the example here works in any Besov space $B_{\infty, q}^{s}$ with $s<1$ and $q \geq 1$. Similarly, one may ask whether the $3 \mathrm{D}$ Euler equations admit an instantaneous loss of regularity in $B_{\infty, q}^{1}$ for any $q>1$. However, this question seems to require a better understanding of the mapping properties of the superposition operator in these spaces than what is currently known.

Acknowledgement. The first author was supported by the James D. Wolfensohn Fund and Friends of the Institute for Advanced Study. The second author was partially supported by JST CREST.

\section{REFERENCES}

[BT] C. Bardos and E. Titi, Loss of smoothness and energy conserving rough weak solutions for the $3 d$ Euler equations, Discrete Contin. Dyn. Syst. 3 (2010).

[CS] A. Cheskidov and R. Shvydkoy, Ill-posedness of basic equations of fluid dynamics in Besov spaces, Proc. A.M.S. 138 (2010), 1059-1067.

[CMT] P. Constantin, A. Majda and E. Tabak, Formation of strong fronts in the 2D quasi-geostrophic thermal active scalar, Nonlinearity 7 (1994), 1495-1533.

[EH] D. Edmunds and D. Haroske, Spaces of Lipschitz type, embeddings and entropy numbers, Rozprawy Mat. 380 (1998), 1-43.

[HK] T. Hmidi and S. Keraani, Global solutions of the super-critical 2D quasi-geostrophic equation in Besov spaces, Adv. Math. 214 (2007), 618-638.

[KNV] A. Kiselev, F. Nazarov and A. Volberg, Global well-posedness for the critical 2D dissipative quasi-geostrophic equation, Invent. math. 167 (2007), 445-453.

[MB] A. Majda and A. Bertozzi, Vorticity and Incompressible Flow, Cambridge Press 2002.

[PP] H.C. Pak and Y.J. Park, Existence of solution for the Euler equations in a critical Besov space, Commun. in PDE 29 (2004).

[Sch] V. Scheffer, An inviscid flow with compact support in space-time, J. Geom. Anal. 3 (1993).

[Shn] A. Shnirelman, Weak solutions with decreasing energy of incompressible Euler equations, Comm. Math. Phys. 210 (2000), 541-603. 
[Tai] M. Taibleson, On the theory of Lipschitz spaces of distributions on Euclidean n-space. I., J. Math. Mech. 13 (1964), II., (ibid) 14 (1965), III., (ibid) 15 (1966).

[Tay] M. Taylor, Tools for PDE, Mathematical Surveys and Monographs vol. 81, Providence 2000.

[Zyg] A. Zygmund, Trigonometric Series, Cambridge Univesity Press 1959.

Institute for Advanced Study, Princeton, NJ 08540, USA and Department of MathEmatics, University of Notre Dame, IN 46556, USA

E-mail address: misiolek@math.ias.edu

Department of Mathematics, Hokkaido University, Sapporo 060-0810, Japan

E-mail address: yoneda@math.sci.hokudai.ac.jp 